\title{
An Organic Nanoparticle based High Signal Amplification Immunoassay with Improvement of Nonspecific Binding
}

\author{
Zhan Wang' and Yuan-Cheng $\mathrm{Cao}^{2 *}$ \\ ${ }^{1}$ Department of Chemical and Environmental Engineering, Wuhan Polytechnic University, Wuhan, Hubei Province, 430023, P. R. China \\ ${ }^{2}$ Key Laboratory of Optoelectronic Chemical Materials and Devices of Ministry of Education, Jianghan University, Wuhan 430056, China
}

\begin{abstract}
A high signal amplification immunoassay method based on Fluorescein Diacetate (FDA) organic nanoparticle was proposed here. FDA nanoparticles were prepared in a ball mill and centrifugation was applied to get the size-uniformed SDS stabilized particle (FDA@SDS). Further SEM images confirmed the particle size was well dispersed in solution and particle size analysis results showed that the particle size $90 \%$ was distributed in the range of $200 \mathrm{~nm}$ to $500 \mathrm{~nm}$. Mass Spectrometry results showed that the antibody can directly attach to the FDA@SDS particles and standard sandwich immunoassay confirmed the bioactivity of the antibody on the FDA@SDS particle surface (FDA@SDS-IgG). And then Zeta potential test results showed that the blocking reagents BSA and Casein can successfully block the unsatisfied sites on the FDA@SDS-IgG surface, which resulted in the improvement of nonspecific binding and stabilization. Further experiments were carried out using the standard immunoassays which showed the analyte signal can be greatly amplified when Casein was used as block buffer in the immunoassays. The limitation of detection was lower than $10 \mathrm{pg} / \mathrm{mL}$, which is 100 times lower than Cy3 or FITC directly labeled antibody. The improved procedure simplifies previously reported protocols and provides a simple high signal amplification immunoassay.
\end{abstract}

Keywords: Fluorescent immunoassays; Organic nanoparticles; Nonspecific binding; Signal amplification; Detection limitation

\section{Introduction}

Some viruses, such as HCV and HIV, can duplicate in very low concentration in the host and so the early infection period diagnosis is very important in such diseases [1-5]. Diagnosis depends on the sensitivity of the detection method and, generally, the lower the detection limit, the shorter the window period between infection and diagnosis [4-7]. And with the development of science in all the fields, loads of new methods and concepts have been designed and developed with high sensitivity and low limit of detection both in serological and molecular approaches [8-13]. Due to the low cost and convenience, labels related immunoassays are the commonly used methods in the clinic practice and thus various formats were developed such as enzyme-linked immunosorbent assays (ELISA), fluorescent immunoassays and radio immunoassays. Among all these immunoassay methods, fluorescent immunoassays are the promising technique both in the laboratory and in the practice with well detection performance.

The detection performance of a fluorescent immunoassay is determined by the number of fluorescent photons emitted per analyte molecule. In order to improve this detection ability, high excitation and high quantum yield fluorophores are pursued [14] or high signal-tonoise materials are applied [7,15-17] or high-load of label molecules systems are designed [11]. These methods are trying to overcome the conventional label system which surfers from the energy transfer and quenching problems when too many of fluorescent molecules are labelled on the targets, while improve the signal amplification either by increasing the fluorescent molecules per analyte (i.e. F/P ratio) or increasing the emitted photons per fluorophore. However, high signal amplification is by far not the only issue the analysts are focusing on in the immunoassays. The lower limitation of detection (LOD) which is affected by nonspecific binding and other aspects is as important as the sensitivity $[1-4,12,13]$. High signal amplification systems increase the analyte signal; however, they will amplify the nonspecific binding and background signals at the same time, especially in the solid-phase fluorescent immunoassays.
Recently, C.P Chan and Dieter Trau et al reported a DSPE-PEG (2000) amine based polyelectrolyte multilayers fluorescein diacetate (FDA) nanocrystals [18-21]; Yvonne Bruemmel et al. [18] studied the influence of different surface modifications on the performance of fluorescent based immunoassays. These methods increase the F/P ratio by releasing fluorescein molecules through the hydrolysis of the FDA nanoparticles after dissolution in DMSO, which undoubtedly can achieve very high sensitivity and very low limit of detection in principle. Beside their study, there are some other aspects should also be considered such as how to deal with the noise signals which might cause by the nonspecific binding or the cross-reactions from the analyte and other proteins in the immunology analytical systems. And this noise signal can also be enlarged by this signal amplification system and it might eliminate the advantages for the related immunoassays.

Here we present a direct antibody adsorpted and blocking reagent coated IgG encapsulated FDA nanoparticles system for fluorescent immunoassay, with the advantages of the low nonspecific binding, easypreparation and high signal amplification, low limit of detection.

\section{Materials and Methods}

\section{Materials}

Fluorescein Diacetate (FDA), Sodium dodecyl sulfate (SDS); Goat anti-human IgG, rabbit anti-goat IgG, human IgG, Cy3 labelled rabbit anti-goat IgG, BSA and PBS were ordered from Sigma. The immunoassays were run on Inverted Fluorescent Microscopy (IX71,

${ }^{*}$ Corresponding author: Yuan-Cheng Cao, Key Laboratory of Optoelectronic Chemical Materials and Devices of Ministry of Education, Jianghan University, Wuhan 430056, China, Tel: 0086 (0) 275125 3048; E-mail: yuancheng.cao@gmail.com

Received April 07, 2014; Accepted June 13, 2014; Published June 17, 2014

Citation: Wang Z, Yuan-Cheng C (2014) An Organic Nanoparticle based High Signal Amplification Immunoassay with Improvement of Nonspecific Binding. J Nanomed Nanotechnol 5: 207. doi: 10.4172/2157-7439.1000207

Copyright: @ 2014 Wang Z, et al. This is an open-access article distributed under the terms of the Creative Commons Attribution License, which permits unrestricted use, distribution, and reproduction in any medium, provided the original author and source are credited. 
<smiles>C=C1OC2(c3ccc(OC(C)=O)cc3Oc3cc(OC(C)=O)ccc32)c2ccccc21</smiles>

Figure 1: Illumination of FDA based immunoassay.

(A)

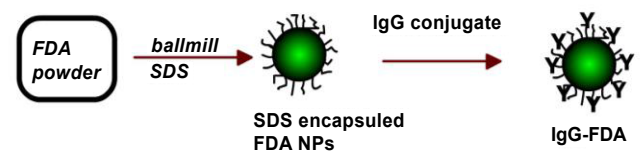

(B)

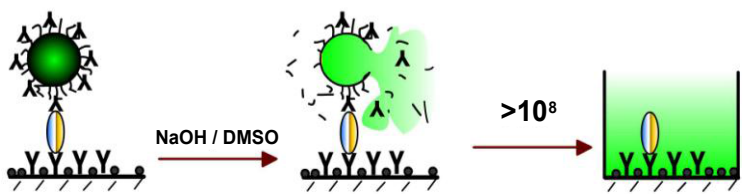

Figure 2: FDA molecule structure.

Olympus) with 96-well plastic-bottom COSTAR plate (catalogue number 3603).

\section{FDA nanoparticles preparation}

$0.2 \mathrm{~g}$ FDA powder and $0.15 \mathrm{~g}$ SDS was added to $10 \mathrm{~mL}$ distilled $\mathrm{H}_{2} \mathrm{O}$, and then the mixture was loaded into the ballmill for 12 hours, at 200 $\mathrm{rpm}$. After that, the solution in the mill was taken out and centrifuged to get the sediment, and then the sediment was dissolved with 5\% SDS. The FDA particle solution was then prepared for SEM by applying it to a surface and coating with $\mathrm{Au}$, and then the coated sample viewed in by SEM (JEOL, Japan).

\section{Preparation of antibody encapsulated FDA@SDS}

$1 \mathrm{~mL}$ of the SDS encapsulated FDA solution was added to $2 \mathrm{~mL} 50$ $\mu \mathrm{g} / \mathrm{mL}$ rabbit anti-goat IgG solution and the mixture was kept shaking for 24 hours before centrifuging at 5,000 rpm to get the FDA@SDS-IgG sediment. Then the sediment was dissolved in $5 \mathrm{~mL}$ PBS solution and divided into 5 tubes $(1 \mathrm{~mL} /$ tube) for further experiments. The process was shown in Figure 1A.

Casein coated FDA@SDS-IgG (FDA@SDS-IgG-Casein): $1 \mathrm{~mL}$ of FDA@SDS-IgG and 1mL of 0.5\% Casein buffer were mixed and centrifuged to get the sediment and the then the sediment was dissolved in PBS solution for further applications.

BSA coated FDA@SDS-IgG (FDA@SDS-IgG-BSA): 1 mL of FDA@ SDS-IgG and $1 \mathrm{~mL}$ of $1 \%$ BSA buffer were mixed and centrifuged to get the sediment, and the then the sediment was dissolved in PBS solution for further applications.

\section{FDA based Immunoassay}

FDA@SDS-IgG particles were applied to standard immunoassays (Figure 1B). Human IgG was immobilized onto the plate surface by overnight incubation and followed by $1 \%$ BSA block; goat anti-human
IgG in series concentrations was loaded into the wells as analyte; and then FDA@SDS-IgG was loaded at 30ml/well and followed by PBS (pH 6.8) buffer washing for 6 times; and then $\mathrm{NaOH} / \mathrm{DMSO}(1: 1, \mathrm{v} / \mathrm{v})$ was loaded into the wells at $50 \mathrm{ml} /$ well; fluorescence was measured after 10 min.

\section{Zeta potential and particle size distribution analysis}

The samples (FDA solution and FDA@SDS-IgG solution) were loaded into the cell for Zeta potential and particle size distribution test on MALVERN Zetasizer Instrument (Zetasizer Nano model). The temperature was set as $25^{\circ} \mathrm{C}$ and the dispersant was water. For the Zeta potential tests, only $\mathrm{pH}$ was adjusted by $1 \mathrm{~N} \mathrm{HCl}$ or $\mathrm{Na}_{2} \mathrm{CO}_{3}$ solution. All the conditions were the same.

\section{Mass spectrometry}

The samples (FDA, FDA@SDS-IgG and IgG solutions) were applied to the plate for the Matrix Assisted Laser Desorption Ionalization Mass Spectrometry (SHIMADU, Kratos Axima CFR ${ }^{+}$MALDI-TOF). Three proteins from laserbiolabs were used for the calibration in these measurements, i.e. Insulin oxidised B chain, Horse myoglobin, Trypsinogen.

\section{Results and Discussion}

\section{FDA@SDS nanoparticles preparation}

Fluorescein diacetate (FDA) is a fluorescein derivative and water insoluble, and it is easy to hydrolyze in the two ester bonds in alkalescent condition, as we can see form the molecule structure (Figure 2). Based on these properties of FDA, an organic nanoparticle related immunoassays can be developed by using ball mill with the help of surfactant SDS solution which makes the FDA water soluble. The interaction between the SDS and FDA particle is mainly by the hydrophobic interaction. As the SDS is an amphiphilic molecular, the hydrophobic alkyl tail contact with the FDA surface and the hydrophilic head faces outside to the aqueous phase. As shown in Figure 1, FDA nanoparticle are easily hydrolysed at high $\mathrm{pH}$ and with the help of DMSO, the SDS encapsulation on the FDA particle is dissolved and thus FDA nanoparticle is released into the $\mathrm{NaOH}$ solution and then FDA is converted into fluorescein which is highly fluorescent.

In the ballmill, the smaller particle size increases the surface/ volume ratio so that more surfactant SDS molecules can be adsorbed to the surface of FDA by hydrophobic interaction, and thus SDS encapsulated FDA nanoparticle was formed (FDA@SDS). As a result, the SDS encapsulation at the surface of FDA particle makes the FDA particles aqueous suspendable. The SEM and particle size analysis were applied for the resultant particles and the results indicated that the FDA@SDS are well dispersed in the solution (Figure 3A) and no aggregations were observed during the prepations. The size distribution is shown in Figure 3B. From these results we can see that the particle size $90 \%$ was distributed in the range of $200 \mathrm{~nm}$ to $500 \mathrm{~nm}$.

Assuming the FDA particle is $250 \mathrm{~nm}$ in sphere, the number of IgG on a single FDA particle in theory is in the range of 2800 to 5600 , depending on the orientations of the absorbed IgG molecules, the percentage of IgG coverage, et al. In our experiments, it is possible the number of the IgG in the range of 100 500 on a single 500nm sized FDA particle, as more protein such BSA and Casein can be farther absorbed. And also it is possible monolayer may be formed. Furthermore, due to the space hinder resistance of the sandwich immunoassays, large number of IgG on single particle is not necessary. 


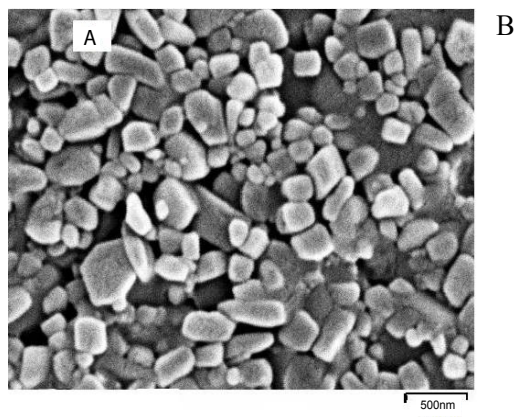

$\mathrm{B}$

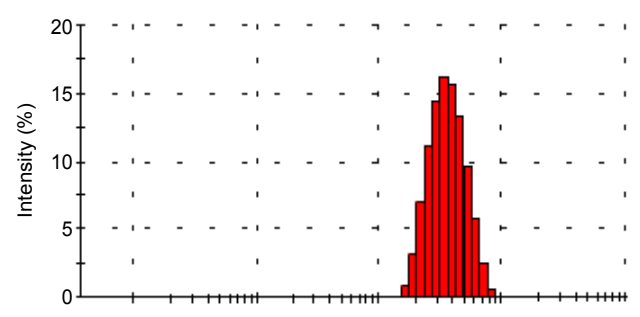

Size (d.nm)

Figure 3: SEM image of FDA nanoparticles $(A)$ and size distribution by intensity (B).
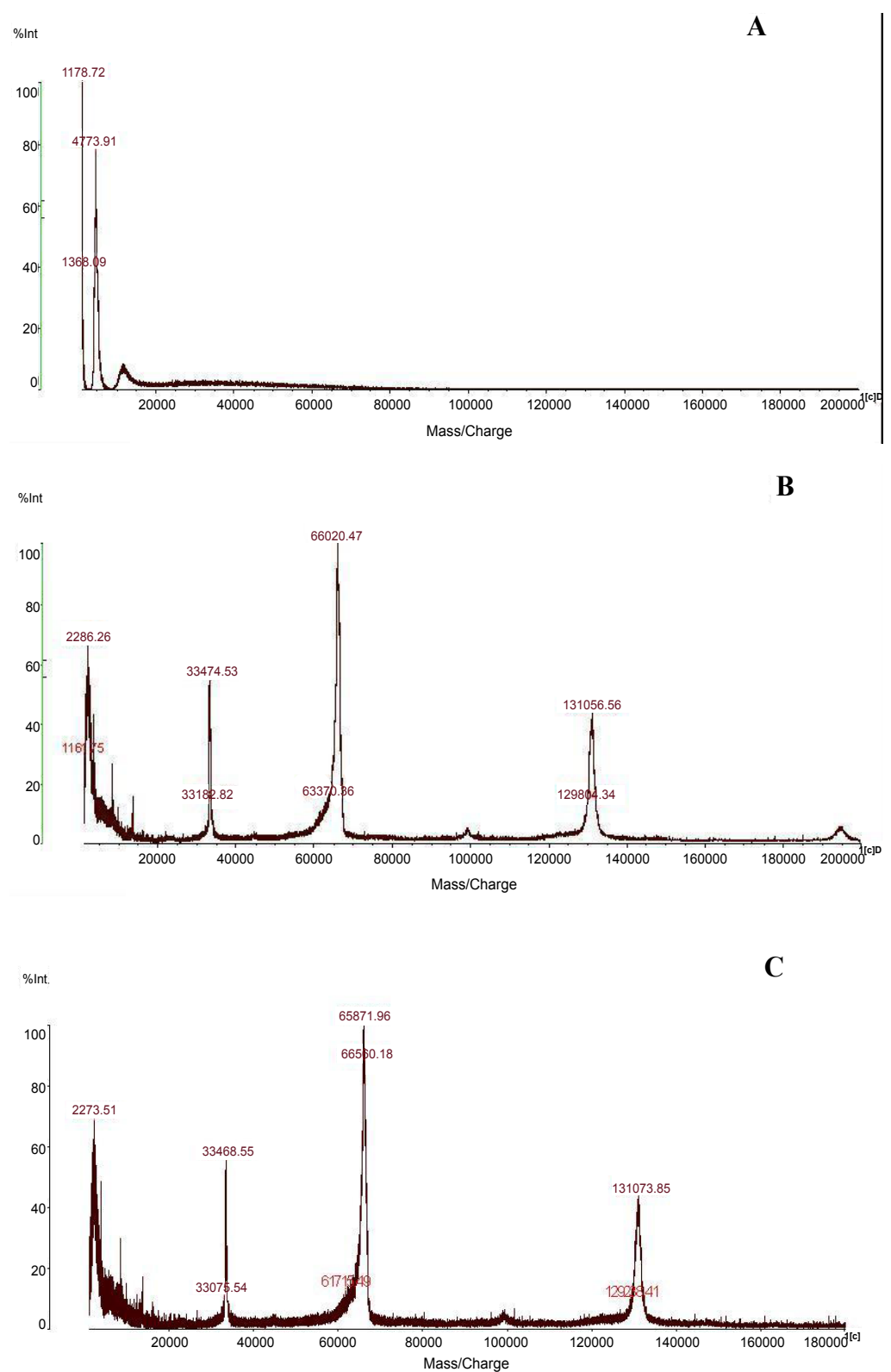

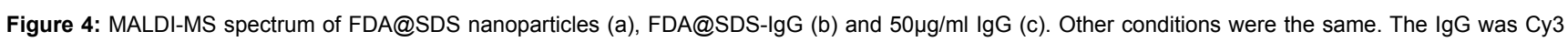
labelled rabbit anti-goat antibody. 


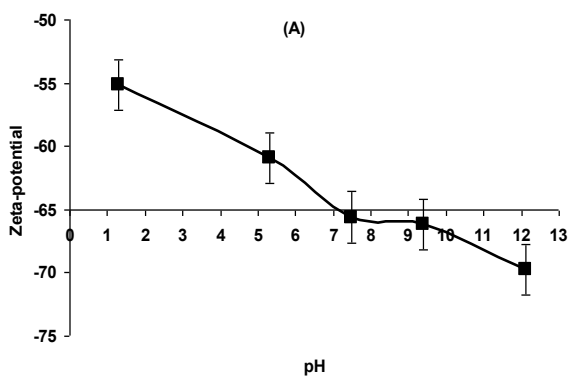

(B)

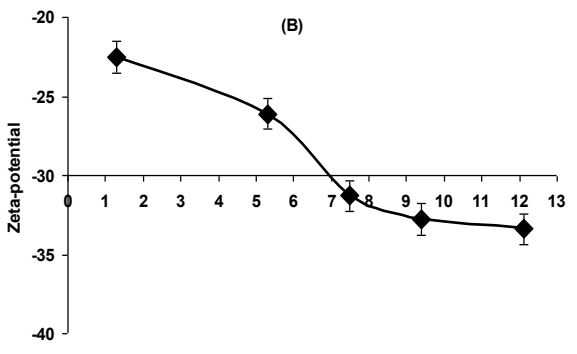

(C)

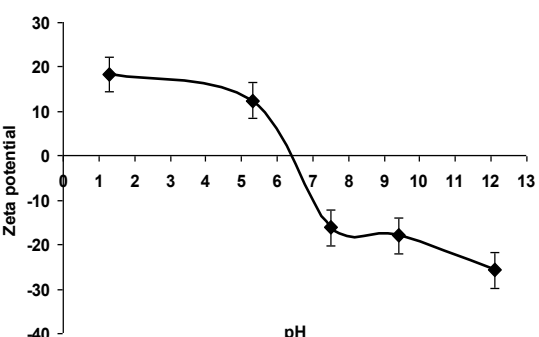

(D)

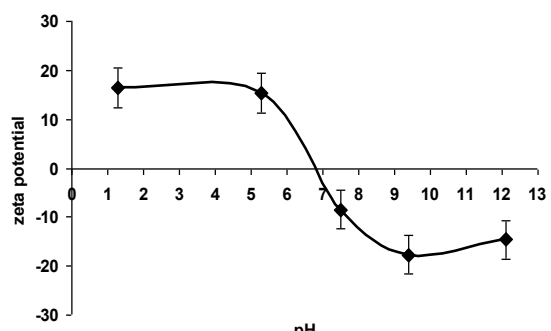

Figure 5: $\xi$-potential of FDA@SDS (A), FDA@SDS-IgG (B), Casein/FDA@SDS-IgG (C) and BSA/FDA@SDS-IgG (D) at different pH. The data were averaged by 5 repeated measurements. All the other conditions were the same.

\section{FDA@SDS and antibody encapsulation and characteristic}

In order to prove that antibody encapsulated on the FDA@SDS particles, MALDI-TOF mass spectrometry was applied to measure the protein on the nanoparticles' surface. The results were shown in Figure 4. In the mass spectrum of FDA@SDS (Figure 4A), there is no ionization peak in high molecule weight $(>10 \mathrm{kDa})$; while in the spectrum of FDA@SDS-IgG (Figure 4B) there are several ionization peaks at $131056.56,66020.47$ and 33474.53 , which is correspond to the ionization of $[\operatorname{IgG}+\mathrm{H}]^{1+}$, $[\operatorname{IgG}+2 \mathrm{H}]^{2+}$ and $[\operatorname{IgG}+3 \mathrm{H}]^{3+}$ respectively. And these ionization peaks can be proved by the pure antibody mass spectrum (Figure 4C) which has the similar results. From the results we can see that the antibody in the solution can be encapsulated to the surface of FDA@SDS surface directly. And following experiments of SEM observations and fluorescent microscopy tests showed the FDA@ SDS-IgG was stable in PBS solution in room temperature without aggregation or hydrolysis. Although antibody can be attached to the FDA@SDS surface, whether the surface becomes satisfied or not is another issue which affects the surface electrostatic property. And the unsatisfied surface charge might increase the non-specific binding during the following immunoassays, because the FDA@SDS-IgG particles may adsorpt to the plate surface which immobilized with proteins by electrostatic interaction. In order to satisfy the unoccupied sites on the FDA@SDS-IgG particles, minimize the non-specific binding and stabilize the FDA@SDS-IgG particles in the solution, commonly used blocking reagents BSA $(66 \mathrm{kDa})$ and Casein $(23 \mathrm{kDa})$ were used to coat the FDA@SDS-IgG particles which can get the resultant FDA@ SDS-IgG-BSA and FDA@SDS-IgG-Casein particles, respectively. The excess of blocking reagent can also satisfy the surface charge after the antibody encapsulation.

To monitor the surface charge, Zeta potential analyzer was applied to measure the charge property of the FDA@SDS, FDA@SDS-IgG, FDA@SDS-IgG-BSA and FDA@SDS-IgG-Casein particle. The results are shown in Figure 5. From the results we can see that both the $\xi$-potential of FDA@SDS-IgG nanoparticles (Figure 5A) and FDA@ SDS nanoparticles (Figure 5B) are much negative either in high $\mathrm{pH}$ value or low $\mathrm{pH}$ value, though it changed a little at different $\mathrm{pH}$ value; however, for the Casein and BSA coated FDA@SDS-IgG nanoparticles (Figure 5C and D), the $\xi$-potential changed steadily from positive to negative when the $\mathrm{pH}$ value was changed from 1.3 to 12.1 .

And by comparing the individual $\xi$-potential at different $\mathrm{pH}$ value, the antibody only satisfied part of the FDA@SDS charged surface while the excess of smaller molecules such as BSA or Casein can satisfy the rest of the charge. The results indicate that this interaction is mainly caused by electrostatic attraction. As in the interaction of SDS molecules and FDA nanoparticles, the dodecyl groups attach to the surface of FDA by hydrophobic interaction, leaving the sulfate groups out of the surface which makes the FDA@SDS nanoparticles in negative charge. These results indicate that the antibody in solution attaches on the surface of FDA@SDS nanoparticle with strong interaction.

\section{Antibody bioactivity test}

Whether the SDS on the FDA nanoparticle surface can denature the encapsulated antibody or not is an important issue in the immunoassays, as the encapsulated antibody has to keep its bioactivities such as antigen-antibody recognition and specificity. In order to test the antibody bioactivities after the encapsulation on the FDA@SDS nanoparticle surface, dose response experiments were carried out when Cy3 labeled rabbit anti goat IgG was encapsulated on the FDA@SDS particles. According to the standard sandwich immunoassay (Figure 6A), human IgG was immobilized on the 96-well plate and goat antihuman IgG was used as analyte which the concentration was from 0,10 , 20 and $50 \mu \mathrm{g} / \mathrm{mL}$, when FDA@SDS-IgG-Cy3 was used as fluorescent reporter, we found that the $\mathrm{Cy} 3$ fluorescent intensity increased as the analyte concentration increased (Figure 6B) and the intensity was linear relation with the analyte concentration (Figure $6 \mathrm{C}$ ) in the range from 0 to $20 \mu \mathrm{g} / \mathrm{mL}$, while it was satisfied when the analyte concentration increased from $20 \mu \mathrm{g} / \mathrm{mL}$ to $50 \mu \mathrm{g} / \mathrm{mL}$. The results showed that the 
Citation: Wang Z, Yuan-Cheng C (2014) An Organic Nanoparticle based High Signal Amplification Immunoassay with Improvement of Nonspecific Binding. J Nanomed Nanotechnol 5: 207. doi: 10.4172/2157-7439.1000207

Page 5 of 7

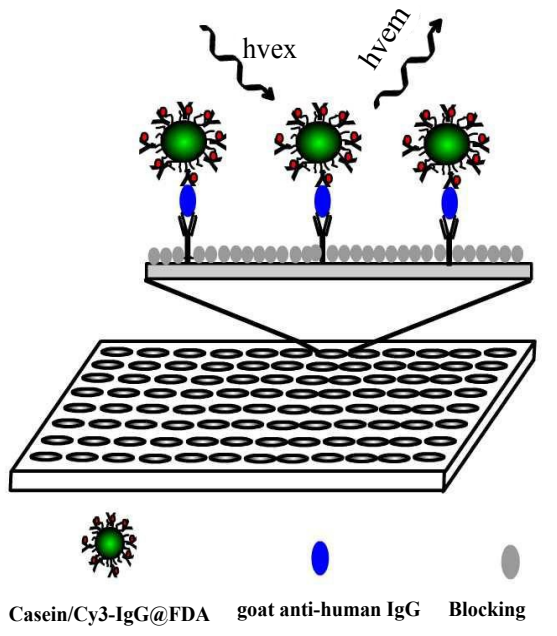

(A)
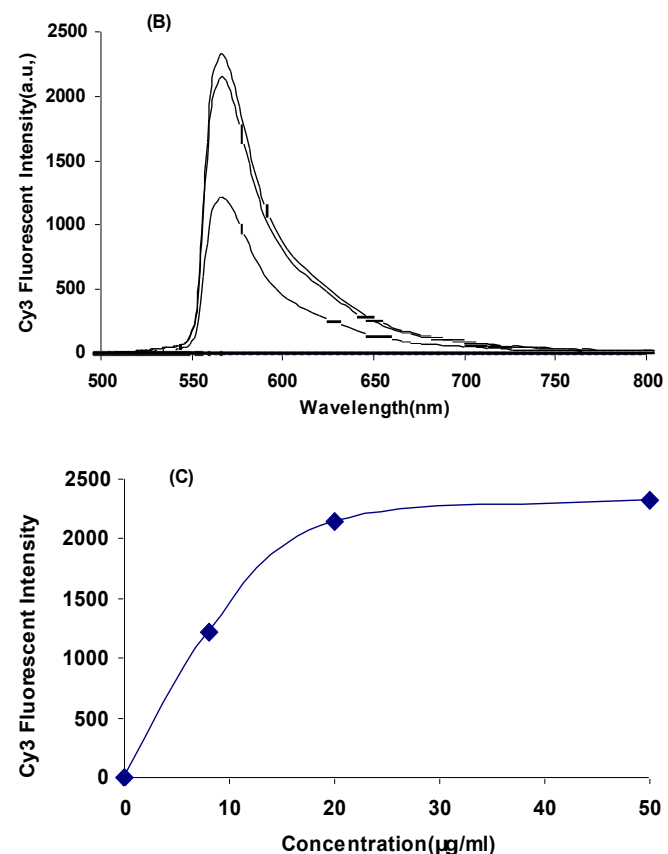

Figure 6: Illustration of encapsulated antibody (FDA@SDS-IgG-Casein) bioactivity test in sandwich immunoassay using Fluorescent Microscopy $(A)$, and the fluorescent spectra of different analyte concentration (B) with dose response curve (C). Exposure time was $500 \mathrm{~ms}$ and with Excitation 480-550nm, Emission 590nm LP filter set.

antibody on the FDA@SDS surface can react with the analyte in a very good dose response which indicates that the encapsulated IgG can keep the bioactivities after the encapsulation. Presumably the SDS molecules and antibody interaction are contributed by the electrostatic interaction without further denature of the antibody. These attached IgGs may in the well-ordered status, i.e. sulfate group outside and lauryl group inside.

\section{FDA@SDS-IgG organic particles based immunoassay}

Nonspecific binding exists in most of the solid-phase immunoassays which has relation with many aspects such as the solid surface property, immunoassay conditions, property of samples and the analysis methods [22,23]. Here, for example, we compared two commonly used blocking buffers, i.e. BSA $(66 \mathrm{kDa})$ and Casein $(23 \mathrm{kDa})$, in the FDA particle based immunoassays; and the results are shown in Table 1. From the table we can see that the FDA@SDS-IgG-Casein particles had better performance in nonspecific binding issue when Casein was used as blocking buffer. We assume this may due to the size of the Casein molecular is much small than BSA, which lead to higher surface protein.

In order to test the analytical performance of the Casein coated FDA@SDS-IgG particles, goat anti-human IgG (used as detection analyte) in series concentration from $50 \mu \mathrm{g} / \mathrm{mL}$ to $10^{-3} \mathrm{ng} / \mathrm{mL}$ were applied to the standard immunoassays. And at the same time, the commonly used $\mathrm{Cy} 3(\mathrm{~F} / \mathrm{P}=3.0 \sim 9.0$, as refer to the Sigma reagent documents) and FITC $(\mathrm{F} / \mathrm{P}=3.0 \sim 8.0$, as refer to the Sigma reagent documents) directly labeled rabbit anti-goat IgG were also applied as comparisons respectively. From the results we can see that the work range for Cy3 directly labelled system was from $100 \mathrm{ng} / \mathrm{ml}$ to $20 \mu \mathrm{g} /$ $\mathrm{mL}$ and could give out a lower limitation of detection (LOD) about $100 \mathrm{ng} / \mathrm{mL}$ (Figure 7) in the experiments. And the results for FITC

\begin{tabular}{|c|c|c|}
\hline Label & BSA Blocking buffer & Casein Blocking buffer \\
\hline System & $\begin{array}{l}\text { NB Fluorescent Intensity } \\
\text { (a.u.) }\end{array}$ & $\begin{array}{l}\text { NB Fluorescent Intensity } \\
\text { (a.u.) }\end{array}$ \\
\hline FDA@SDS-IgG-Casein & $835 \pm 46.6$ & $146.5 \pm 45$ \\
\hline FDA@SDS-IgG-BSA & $1506 \pm 414.8$ & $418.3 \pm 46.2$ \\
\hline
\end{tabular}

(1) The Filter set was $470 \mathrm{~nm}$ excitation with 515 Long pass emission; the exposure time was 100 ms with the intensifier profile Math DC 500 and Cath - 50 .

(2) NB: Nonspecific Binding (Unit: a.u.).

Table 1: Nonspecific binding in different labels and blocking buffers system ${ }^{(1)}(n=4)$
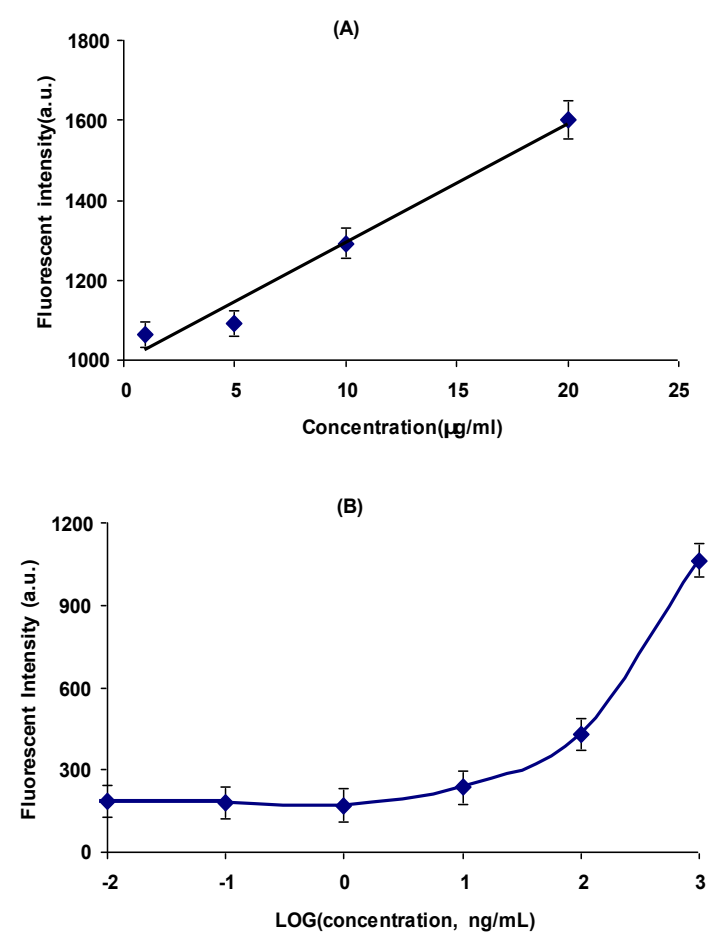

Figure 7: Cy3 directly labeled antibody dose response $(A)$ and lower limit of detection in sandwich immunoassay (B). Cy3 labeled rabbit anti-goat IgG was used and the exposure time was $500 \mathrm{~ms}$, all the other conditions were the same. 
Citation: Wang Z, Yuan-Cheng C (2014) An Organic Nanoparticle based High Signal Amplification Immunoassay with Improvement of Nonspecific Binding. J Nanomed Nanotechnol 5: 207. doi: 10.4172/2157-7439.1000207

Page 6 of 7

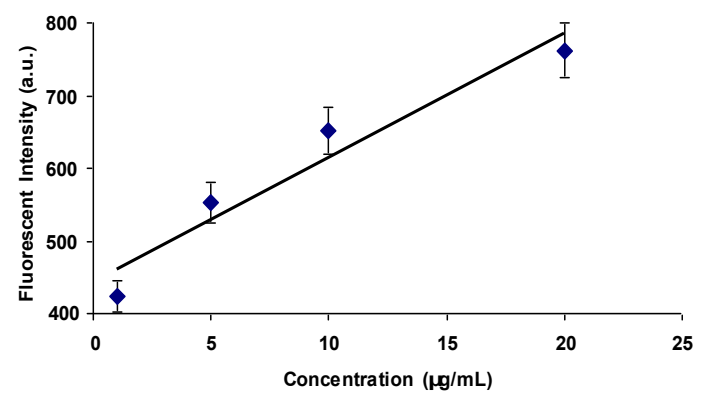

(B)

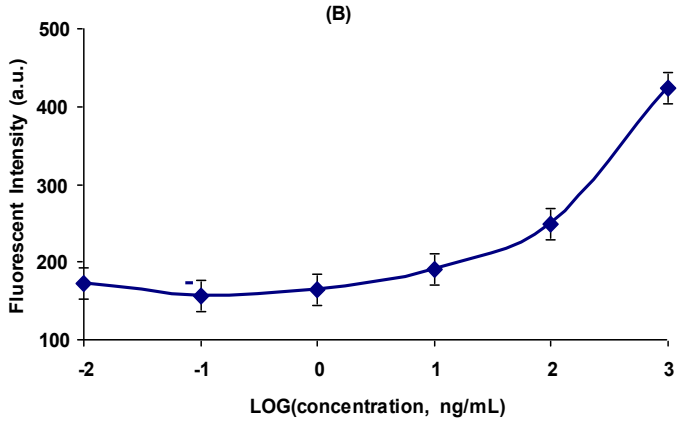

Figure 8: FITC directly labeled antibody dose response (A) and lower limit of detection in sandwich immunoassay (B). FITC labeled rabbit anti-goat lgG was used and the exposure time was $500 \mathrm{~ms}$, all the other conditions were the same.
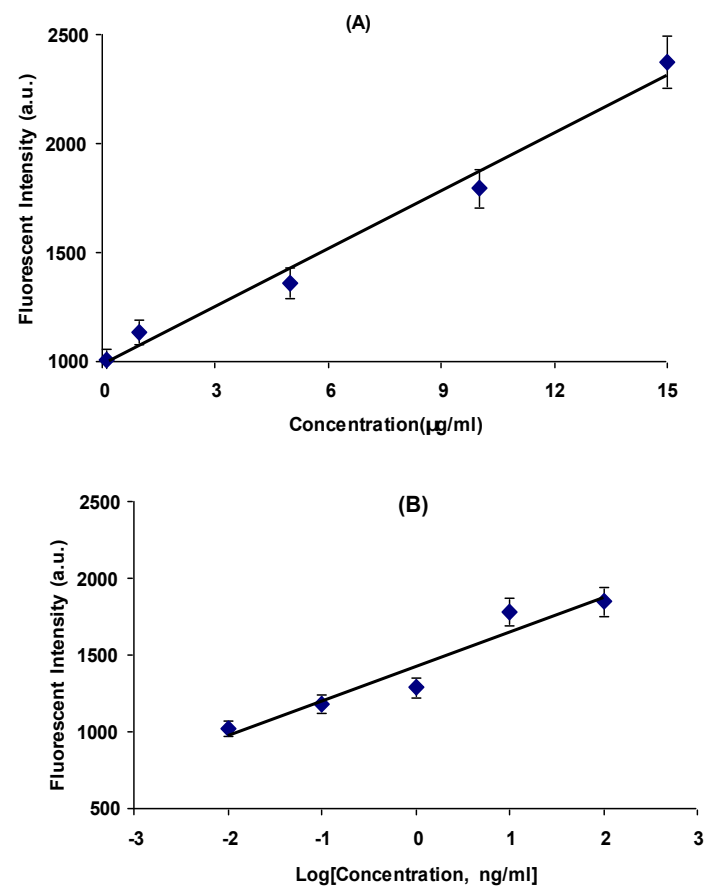

Figure 9: FDA@SDS-IgG-Casein based immunoassay for the dose response (A) and lower limit of detection (B). Rabbit anti-goat IgG was used and the exposure time was $100 \mathrm{~ms}$.

directly labeled system gave out the similar results, as shown in Figure 8 . For both of these two dyes directly labeled antibody, if the detection analyte concentration is lower than $100 \mathrm{ng} / \mathrm{ml}$, the analyte signal is not distinguishable from nonspecific binding and background signal. For the FDA@SDS-IgG-Casein particles, the higher limitation of detection could only reach to $15 \mu \mathrm{g} / \mathrm{mL}$ under the same conditions (Figure 9A), and this is because the size of coated FDA particle used as labeled antibody is much larger than the dye directly labeled antibody. But the LOD was not reach at the concentration of $10^{-2} \mathrm{ng} / \mathrm{mL}$ in our experiments (Figure 9B), and that is to say, the detectable concentration is about $10^{-5.5} \mu \mathrm{g} / \mathrm{mL}$ which is 67 atto $\mathrm{mol} / \mathrm{mL}$ [24]. Therefore, from the results we can see that the FDA@SDS-IgG-Casein particles have advantages in the very low analyte concentration samples and this is very important for the clinic diagnostics for the virus such HCV, HIV, which can greatly shorten the "detection window". This method is promising to diagnose in the early weeks of virus infections.

\section{Conclusions}

FDA nanoparticles can be successfully made by ball mill in SDS surfactant solution. The size distribution can be uniformed further by speed-gradient centrifugation. And the SDS encapsulated FDA nanoparticles can be further encapsulated with small blocking protein molecules such as casein to satisfy the surface charge by monitoring the zeta potential. And this process can also make the FDA particles more stable in the solution without aggregation and self-dehydrate. The blocking proteins can effectively eliminate the nonspecific binding which caused by the electrostatic absorption in the applied immunoassays. The FDA@SDS-IgG-Casein based immunoassay can achieve high signal amplification and low analyte detection. By further reducing nonspecific binding, the expected detectable lower concentration should be lower than $10 \mathrm{pg} / \mathrm{mL}$.

\section{References}

1. Berger A, Rabenau H, Allwinn R, Doerr HW (2008) Evaluation of the new ARCHITECT anti-HCV screening test under routine laboratory conditions. J Clin Virol 43: 158-161.

2. Vermeersch P, Van Ranst M, Lagrou K (2008) Validation of a strategy for HCV antibody testing with two enzyme immunoassays in a routine clinical laboratory. J Clin Virol 42: 394-398.

3. Biliotti E, Kondili LA, Furlan C, Ferretti G, Zacharia S, et al. (2008) Acute hepatitis $B$ in patients with or without underlying chronic HCV infection. J Infect 57: 152-157.

4. Desbois D, Vaghefi P, Savary J, Dussaix E, Roque-Afonso AM (2008) Sensitivity of a rapid immuno-chromatographic test for hepatitis $\mathrm{C}$ antibodies detection. $J$ Clin Virol 41: 129-133.

5. Sulkowski MS (2008) Viral hepatitis and HIV coinfection. J Hepatol 48: 353367.

6. Goodman WG, Huang ZH, Robinson GE, Strambi C, Strambi A (1993) Comparison of two juvenile hormone radioimmunoassays. Arch Insect Biochem Physiol 23: 147-152.

7. Natalia CT, Gao Z (2006) Nanoparticles in biomolecular detection. Nanotoday 1: 28-37.

8. Cao C, Shi C, Li P, Tong Y, Ma Q (1996) Diagnosis of hepatitis C virus (HCV) infection by antigen-capturing ELISA. Clin Diagn Virol 6: 137-145.

9. De Cock L, Hutse V, Verhaegen E, Quoilin S, Vandenberghe H, et al. (2004) Detection of HCV antibodies in oral fluid. J Virol Methods 122: 179-183.

10. Schröter M, Zöllner B, Schäfer P, Laufs R, Feucht HH (2001) Comparison of three HCV genotyping assays: a serological method as a reliable and inexpensive alternative to PCR based assays. J Clin Virol 23: 57-63.

11. Takashi M, Fumiko S, Junko T (1994) Comparison of HCV core antigen activity by ELISA and amount of HCV RNA by branched DNA assay. Int Hepatol Comm 2: $175-177$.

12. Khlebtsov B, Khlebtsov N (2008) Enhanced solid-phase immunoassay using gold nanoshells: effect of nanoparticle optical properties. Nanotechnology 19 : 435703. 
Citation: Wang Z, Yuan-Cheng C (2014) An Organic Nanoparticle based High Signal Amplification Immunoassay with Improvement of Nonspecific Binding. J Nanomed Nanotechnol 5: 207. doi: 10.4172/2157-7439.1000207

13. Kingsmore SF, Patel DD (2003) Multiplexed protein profiling on antibody-based microarrays by rolling circle amplification. Curr Opin Biotechnol 14: 74-81.

14. Hua XF, Liu TC, Cao YC, Liu B, Wang HQ, et al. (2006) Characterization of the coupling of quantum dots and immunoglobulin antibodies. Anal Bioanal Chem 386: 1665-1671.

15. Cao YC1, Liu TC, Hua XF, Zhu XX, Wang HQ, et al. (2006) Quantum dot optical encoded polystyrene beads for DNA detection. J Biomed Opt 11: 054025.

16. Cao YC, Huang ZL, Liu TC, Wang HQ, Zhu XX, et al. (2006) Preparation of silica encapsulated quantum dot encoded beads for multiplex assay and its properties. Anal Biochem 351: 193-200.

17. Cao YC, Wang Z, Wang HQ, Wang JH, Hua XF, et al. (2009) Enhanced optical property of Au coated polystyrene beads for multi-color quantum dots encoding. J Nanosci Nanotechnol 9: 1778-1784.

18. Bruemmel Y, Chan CP, Renneberg R, Thuenemann A, Seydack M (2004) On the influence of different surfaces in nano- and submicrometer particle based fluorescence immunoassays. Langmuir 20: 9371-9379.
19. Chan CP, Bruemmel Y, Seydack M, Sin KK, Wong LW, et al. (2004) Nanocrystal biolabels with releasable fluorophores for immunoassays. Anal Chem 76: 3638 3645 .

20. Trau D, Yang W, Seydack M, Caruso F, Yu NT, et al. (2002) Nanoencapsulated microcrystalline particles for superamplified biochemical assays. Anal Chem 74: 5480-5486

21. Yang W, Trau D, Renneberg R, Yu NT, Caruso F (2001) Layer-byLayer Construction of Novel Biofunctional Fluorescent Microparticles for Immunoassay Applications. J Colloid Interface Sci 234: 356-362.

22. Wakayama J, Sekiguchi H, Akanuma S, Ohtani T, Sugiyama S (2008) Methods for reducing nonspecific interaction in antibody-antigen assay via atomic force microscopy. Anal Biochem 380: 51-58.

23. Pei R, Cui X, Yang X, Wang E (2000) Real-time immunoassay of antibody activity in serum by surface plasmon resonance biosensor. Talanta 53: 481-488.

24. Daniel CH (2003) Quantitative Chemical Analysis. (6thedn), W.H.Freeman and company, New York: 66-79. 